\title{
Application of "Monarch, Minister, Assistant and Envoy" Principle to Rheumatoid Arthritis Management
}

\author{
Yong Chen ${ }^{1}$, Ji-nan Zheng ${ }^{2}$, Jun Fang ${ }^{2}$, Shen-guang Li $^{3}$, Jiang-long Guan ${ }^{1}$ and Pei- \\ wei Yang ${ }^{2 *}$ \\ ${ }^{1}$ Rheumatologic Department, Huadong Hospital, Fudan University Shanghai 200040 \\ ${ }^{2}$ Orthopedics department, The 180th Hospital of PLA, Quanzhou Fujian 362000 China \\ ${ }^{3}$ Rheumatologic Department, The first affiliated hospital of Chinese PLA general hospital, Beijing \\ 100048
}

Received: March 8, 2017; Accepted: June 23, 2017; Published: September 05, 2017

*Corresponding author: Guan Jianglong, Rheumatologic Department, Huadong Hospital, Fudan University Shanghai 200040,

Email: guanjianlong@medmail.com.cn

\begin{abstract}
This paper illustrates "monarch, minister, assistant and envoy" principle of traditional Chinese medicine through clinical treatment guideline of rheumatoid arthritis. The conception can promote the precise treatment in rheumatoid arthritis management, and prevent side effects of drugs consciously. To applicant the "monarch, minister, assistant and envoy" principle to direct clinical drug options for rheumatoid arthritis may facilitate a more flexible, safer, and less drug intake.
\end{abstract}

Keywords: Rheumatoid arthritis; Drug; Side effect; Traditional Chinese Medicine; Excessive treatment

\section{Introduction}

Rheumatoid arthritis is a long-lasting autoimmune disease in clinic, which typically results in warm, swollen and painful joints, the waist and hands are most commonly involved. The progression of the disease can cause irreversible deformity. It can also affect other parts of the organs or systems, and result in anemia, fatigue, inflammation around the lungs, atherosclerosis, and inflammation around the heart [1-3]. Besides of function loss of extremities, diverse complications especially infection is risk factors for early death in these patients [4]. The prevalence of rheumatoid arthritis is around $0.5 \%-1 \%$ in advanced countries, some 49,000 people died of rheumatoid arthritis in 2010 worldwide $[5,6]$. Due to the high incidence and disability and mortality rates, along with the huge medical research investment, variety of drugs has been exploited providing sundry options for clinical management. Excessive treatment, side effects from drugs and overrating benefits from pharmacies are issues needs alertness.

Obviously, it is not personalized enough to solve complains of each patient through frigidly execution of authoritative guidelines or recommendations published. Is there a way or principle of selecting drugs for more accurate treatment for rheumatoid arthritis patients? Traditional Chinese Medicine (TCM) gained afflatus from war that similar principles dominate in both military and medicine that is to prescribe drugs according to military action strategies or country governance $[7,8]$. For example, it is recorded in Yellow Emperor (Huangdi Neijing) that army force should be prepared to eliminate invasion, it would be too late to establish military force when an invasion is breaking out. Medication is prescribed to cure diseases; and medicine should not be given delayed until illness progressed. Military strategies and medicine share a same truth. Another quotation from Military Base (Bin lei) says, an eminent general conducts troops resembles an eminent doctor applies of drugs. It needs to change drugs proactively if an illness state changed instantaneously. However, if an illness fluctuated while prescription revising not followed, none of the diseases could be cured. It is summarized the roles of drugs in a prescription formula as Jun (Monarch), Chen (Minister), Zuo (Assistant) and Shi (Envoy) in TCM theories, which we think could be enlighten our modern medical recipes for rheumatoid arthritis patients as well.

\section{Conceptions of Jun, Chen, Zuo and Shi}

Jun, Chen, Zuo and Shi originally means Monarch, Minister, Assistant and Envoy four types of people in a government, and currently is used to expound various herbs of different functions in a TCM prescription formula. The theory was first recorded in Shennong Bencao Jing (Variorum of Shennong's Classic of Materia Medica), and was recorded and discussed by following medical literatures of successive dynasties in Chinese history, such as Yellow Emperor, Treatise on Spleen and Stomach and Medicine Origin etc.. Jun, Chen, Zuo and Shi is one of the most basic theories in TCM for prescribe herbal medical formula. 
In a formula, the monarch drugs called Jun, which refer to the drugs that target the disease or main symptoms suffered; it reflects the main direction the prescription treating. Jun is an indispensable drug in a formula. Chen drugs are used to assistant Jun to enhance the effectiveness for the main disease or symptoms. Zuo means assisting drugs, which may show roles in three aspects. Firstly, they used to treat subordinated diseases. Secondly, they help to relive or eliminate toxicities or side effects caused by Jun and Chen drugs. Thirdly, they were used to exhibit opposite effects of Jun drugs nevertheless are necessary for certain situation for a patient. Shi is translated to envoy, refers to drugs guiding other drugs in a formula to achieve organs involved, Jingluo attacked or disease location. Shi also helps to harmonize drugs in a formula to eliminate pathogens.

In this Chinese classical literature, it describes 108 plucky men organize themselves to revolt the enslaving from corrupt government. Each person with its talent and has a corresponding role in the team to fight, which shares a same truth in a prescription of multiple drugs with different but should have a well organized function for disease (Figure 1).

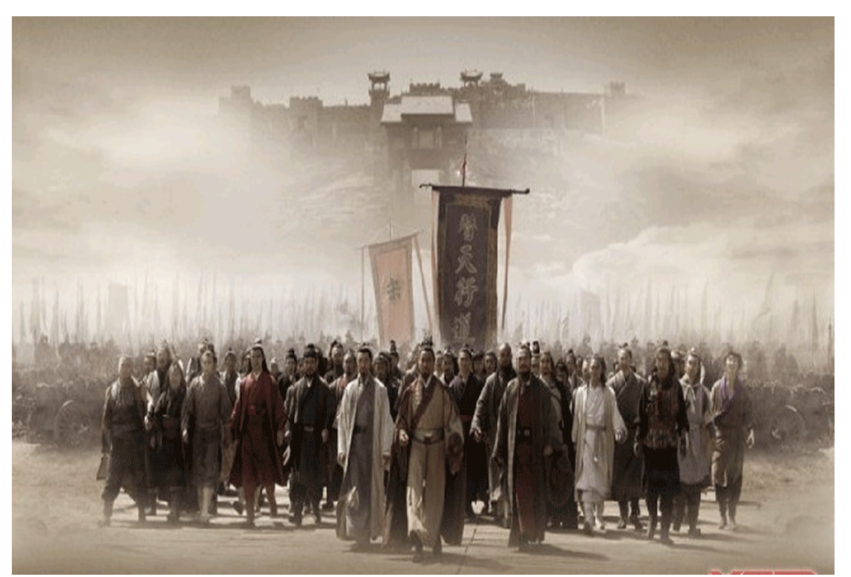

Figure1: A poster of Water Margin

\section{Medications for RA classified according to Jun, Chen, Zuo, Shi}

Emergence in the 1970s, the term Disease Modifying AntiRheumatic Drugs (DMARDs) is a category of otherwise unrelated drugs defined by their use in rheumatoid arthritis to slow down disease progression of joints erosion, to relieve synovial inflammation, and to improve body function [9]. According to 2015 American College of Rheumatology (ACR) guideline for the treatment of rheumatoid arthritis [10], no matter early diagnosed or established RA, from low diseases to moderate and high disease activity, DMARD monotherapy is regarded as the first line option. In circumstance that patients with inflammation relapse or treatment failure with DMARD monotherapy, options of combination of DMARDs or DMARDs added to glucocorticoids or TNFi are recommended. In a word, DMARDs are irreplaceable medication and the main effective drugs for RA management. So
DMARDs are regarded as Jun drugs. If single DMARD or combined DMARDs cannot control the symptoms, or the disease still relapses, low dosage of glucocorticoids is recommended. If the disease activity is still in middle to high degree, biological agents would be considered for these intractable cases. Glucocorticoids and biological agents are used to strengthen the effectiveness on the basis of DMARDs, so they are assimilated to Chen drugs.

Methotrexate is the first option of DMARDs. The mechanisms of methotrexate in treatment of rheumatoid arthritis including inhibiting neutrophil chemotaxis [13], down regulating pro-inflammatory cytokines such as TNF- $\alpha$, IFN, IL-6 et al [14]. Methotrexate has many side effects; the most commonly seen is inhibiting synthesis of folic acid. So taking folic acid or folinic acid next day after intake of methotrexate is recommended, by which to prevent gastrointestinal side effects, liver damage, anemia and leucopenia $[15,16]$. Obviously, folic acid is a Zuo drug for methotrexate to relive or eliminate toxicities or side effects caused. Similarly, side effects caused by all types of DMARDs or glucocorticoids, should be avoided through withdrawal the drug, or combining with medication to anti side effects. As most drugs may have side effects for gastrointestinal tract, and peptic ulcer is 2 times of general in rheumatoid arthritis population [17]. So a gastric mucosal protective agent is recommended to rheumatoid arthritis patients for regular taking.

The conception of Shi (envoy drug) is far-fetched if introduced to rheumatoid arthritis treatment, so it is not discussed in this article.

\section{Benefits of utilizing Jun, Chen, Zuo, Shi principle Administer drugs flexibly}

TCM has a profound acquaintance in the development of a disease with yin-yang and Yi (changing) theories, which contain a full-order system of changing. Treatment based on syndrome differentiation was run through the full course of diagnosis and treatment, progression and prognosis. The conception has distinguished advantages than today's medicine. During active phase of rheumatoid arthritis with joints pain, application of glucocorticoids, which binding to both monomeric and dimeric glucocorticoid receptor or injecting which into particular cavity directly would result in anti-inflammatory effects so as to relieve symptoms of pain and swollen rapidly [18]. That is to say we regard glucocorticoids as Jun drugs during active phase of rheumatoid arthritis. Rheumatoid arthritis can involve respiratory system, cardiovascular system, nerve system, liver, kidney and eye and causes various complications [5]. In a certain disease period or a specific patient, a complication may have more threatening risk for life, and became the major symptom for the patient. The drugs target the fatal complication regarded as Jun drugs, while DMARDs have a Chen status. These instances indicate the utilization of Jun, Chen, Zuo, Shi could pave a flexible way of selecting drugs. And ultimately, the personalized accurate treatment option could be achieved. 


\section{Administer drugs safely}

Supplement of folic acid as Zuo during MTX is taken; supplement of vitamin $\mathrm{D}$ and calcium as Zuo during glucocorticoids is taken. Recheck of blood counts, liver and kidney function regularly while taking DMARDs, TNFi or other immunosuppressive agents, to prevent infection. Timely aware, and stop or replace the drugs if side effects appeared. So if we bear the conception of Jun, Chen, Zuo, Shi in mind, a safer medication application could be achieved.

\section{Prescribe less medications}

As rheumatoid arthritis is a systemic chronic disorder, clinical individual patients may have various complications. And under current medical system, more specifically, there is an interdependent relation between doctors and pharmaceutical manufacturers, patients exposed to the risk of excessive medical treatment vulnerably. However, the increased risk of side effects is accompanied by each drug added. Sometimes, the so called Evidence Based Medicine is an authoritative tool of smiling hurt prescribed from doctors' HIS (hospital information system). Few clinicians or pharmacists can fully illustrate the metabolic process and interference between drugs clearly if a patient taking more than 5 drugs. During clinical practice, we find that to prescribe medications under Jun, Chen, Zuo, Shi theory will not increase the medications chosen, on the contrary, it is a conception for prescribing more concisely in rheumatoid arthritis and other diseases as well.

\section{Conclusions and prospects}

Rheumatoid arthritis is characterized by synovial inflammation and hyperplasia ("swelling"), autoantibody production (rheumatoid factor and Anti-Citrullinated Protein Antibody [ACPA]), cartilage and bone destruction ("deformity"), as well as systemic features, including cardiovascular, pulmonary, psychological, and skeletal disorders [4]. The abundant basic researches in rheumatoid arthritis have accelerated new drugs for the disease, which has resulted in a marked improvement in clinical outcomes. The current treatment strategy can reflect this achievement, once been diagnosed, and the disease activity is accessed, positive treatment would be followed. Through there is no treatment cures rheumatoid arthritis; the therapeutic goals are a remission of symptoms involving the joints, a return of full function, and the maintenance of remission. Unfortunately, there is still a part of patients received partial or failed of remission, sustained remission is rarely achieved and most patients requires ongoing pharmacologic therapy, and mortality rate is higher among patients than healthy persons, and systemic complications remain a major challenge. Therefore, rheumatologists need to be carefully and fully understand the holistic condition of each individual patient, apply of Evidence Based Medicine and clinical guidelines rationally. Traditional Chinese medicine, which has been inherited for thousands of years, apply of state governance and military tactics to medical treatment figuratively, and produce a Jun, Chen, Zuo, Shi combination principle of prescription.
We do not think it is outdated if applied to modern medicine. In this paper, we assimilate DMARDs to Jun, glucocorticoids and biological agents were assimilated to Chen, while drugs confronting side effects caused by Jun and Chen including folic acid, drugs for protecting liver and stomach, calcium and vitamin D supplements were assimilated to Zuo. Under such instruction, drugs could be selected concisely and safely. Doctors need observe patients' disease activity, complications continuously, and arrange the status of drugs according to severity and priority for each patient. For example, in an acute phase, glucocorticoids may play the role of Jun to relieve joints pain in a short time. Or for a rheumatoid arthritis patient with acute onset of coronary heart disease, antithrombotic agents may play the role of Jun. Under this principle, doctor can administer drugs flexibly and personalized, deepen the preventive consciousness for side effects, so as to prescribe rationally and avoid of overtreatment.

Rheumatoid arthritis is still regarded as hazardous diseases through there are many drugs for choosing, published guidelines for following. This article discussed the Jun, Chen, Zuo, Shi principle for guidelines of modern medicines with insufficient instances. However, there is no corresponding part of Shi (envoy) drugs in modern drugs, and to category drugs according to Jun, Chen, Zuo, Shi based on efficacy, utility, toxicity need to be further investigated.

\section{References}

1. Firestein GS. Evolving concept of rheumatoid arthritis. Nature. 2003;423:356-361.

2. Lake F, Proudman S. Rheumatoid arthritis and lung disease: from mechanisms to a practical approach. Seminars in Respiratory \& Critical Care Medicine. 2014;35(2):222-238. doi: 10.1055/s-00341371542

3. Cynthia Crowson S, Katherine Liao P, John Davis M, Daniel Solomon H, Eric Matteson L, Keith Knutson L. Rheumatoid Arthritis and Cardiovascular Disease. American Heart Journal. 2013;166:622-628.

4. Mcinnes IB, Schett G. The Pathogenesis of Rheumatoid Arthritis. New England Journal of Medicine. 2011;365:2205-2219. doi: 10.1056/ NEJMra1004965

5. Scott DL, Wolfe F, Huizinga TW. Rheumatoid arthritis. Lancet. 2010;376:1094-1108. doi:10.1016/S0140-6736(10)60826-4

6. Lozano R, Naghavi M, Foreman K, Lim S, Shibuya K, et al. Global and regional mortality from 235 causes of death for 20 age groups in 1990 and 2010: a systematic analysis for the Global Burden of Disease Study 2010. Lancet. 2012;380:2095-2128. doi: 10.1016/S01406736(12)61728-0

7. Wang JJ. Discussion on Concept of "the Monarch and His Subjects,Assistant and Envoy" and Applied Principle from Documents. China Journal of Basic Medicine in Traditional Chinese Medicine. 2004:19-46.

8. Dong Y, Lin Q, Zhang G. Relationship between Ancient War and Traditional Chinese Medicine. Journal of Traditional Chinese Medicine. 2014;55:369-372.

9. Buer JK. A history of the term "DMARD". INFLAMMOPHARMACOLOGY 2015;23:163-171. 
10. Singh JA, Saag KG, Bridges SJ, Akl EA, Bannuru RR, et al. 2015 American College of Rheumatology Guideline for the Treatment of Rheumatoid Arthritis. Arthritis Rheumatol. 2016;68:1-26.

11. Kraan MC, Koster BMD, Elferink JGR, Post WJ, Breedveld FC, Tak PP. Inhibition of neutrophil migration soon after initiation of treatment with leflunomide or methotrexate in patients with rheumatoid arthritis: Findings in a prospective, randomized, double-blind clinical trial in fifteen patients. ARTHRITIS \& RHEUMATOLOGY. 2000;43:14881495.

12. Riksen NP, Barrera P, Broek PHHV, Riel PLCM, Smits P, et al. Methotrexate modulates the kinetics of adenosine in humans in vivo. Annals of the Rheumatic Diseases. 2006;65:465-470.

13. Shea B, Swinden MV, Ghogomu ET, Ortiz Z, Katchamart W, et al. Folic acid and folinic acid for reducing side effects in patients receiving methotrexate for rheumatoid arthritis. Journal of Rheumatology. 2014;41:1049-1060
14. Whittle SL, Hughes RA. Folate supplementation and methotrexate treatment in rheumatoid arthritis: a review. Rheumatology (Oxford). 2004;43:267-271.

15.Atwater EC, Mongan ES, Wieche DR, Jacox RF. Peptic ulcer and rheumatoid arthritis: a prospective study. Arch Intern Med. 1965;115:184-189.

16. Baschant U, Lane NE, Tuckermann J. The multiple facets of glucocorticoid action in rheumatoid arthritis. Nature Reviews Rheumatology. 2012;8:645-655. doi: 10.1038/nrrheum.2012.166 